Wright State University

CORE Scholar

\title{
Impact of tDCS on Performance and Learning of Target Detection: Interaction with Stimulus Characteristics and Experimental Design
}

Brian A. Coffman

M. C. Trumbo

R. A. Flores

Christopher M. Garcia

A. J. van der Merwe

See next page for additional authors

Follow this and additional works at: https://corescholar.libraries.wright.edu/wsri

Part of the Neuroscience and Neurobiology Commons

\section{Repository Citation}

Coffman, B. A., Trumbo, M. C., Flores, R. A., Garcia, C. M., van der Merwe, A. J., Wassermann, E. M., Weisend, M. P., \& Clark, V. P. (2012). Impact of tDCS on Performance and Learning of Target Detection: Interaction with Stimulus Characteristics and Experimental Design. Neuropsychologia, 50 (7), 1594-1602. https://corescholar.libraries.wright.edu/wsri/5

This Article is brought to you for free and open access by the Wright State Research Institute at CORE Scholar. It has been accepted for inclusion in Wright State Research Institute Publications by an authorized administrator of CORE Scholar. For more information, please contact library-corescholar@wright.edu. 


\section{Authors}

Brian A. Coffman, M. C. Trumbo, R. A. Flores, Christopher M. Garcia, A. J. van der Merwe, Eric M. Wassermann, Michael Patrick Weisend, and Vincent P. Clark 


\title{
Impact of tDCS on performance and learning of target detection: Interaction with
} stimulus characteristics and experimental design

\author{
B.A. Coffman a,b , M.C. Trumbo ${ }^{b}$, R.A. Flores ${ }^{\text {a }}$, C.M. Garcia ${ }^{\text {a,b }}{ }^{\text {, A.J. van der Merwe }}{ }^{\text {a }}$, E.M. \\ Wassermann ${ }^{\mathrm{d}}$, \\ M.P. Weisend ${ }^{\mathrm{a}}$, V.P. Clark ${ }^{\mathrm{a}, \mathrm{b}, \mathrm{c}, *}$ \\ a The Mind Research Network and Lovelace Biomedical and Environmental Research Institute, \\ Albuquerque, NM, United States \\ b Psychology, University of New Mexico, Albuquerque, NM, United States \\ c Neuroscience, University of New Mexico, Albuquerque, NM, United States \\ d National Institute of Neurological Disorders and Stroke, NIH, Bethesda, MD, United States
}

\begin{abstract}
We have previously found that transcranial direct current stimulation (tDCS) over right inferior frontal cortex (RIFC) enhances performance during learning of a difficult visual target detection task (Clark et al., 2012). In order to examine the cognitive mechanisms of tDCS that lead to enhanced performance, here we analyzed its differential effects on responses to stimuli that varied by repetition and target presence, differences related to expectancy by comparing performance in single- and double-blind task designs, and individual differences in skin stimulation and mood. Participants were trained for $1 \mathrm{~h}$ to detect target objects hidden in a complex virtual environment, while anodal tDCS was applied over RIFC at $0.1 \mathrm{~mA}$ or $2.0 \mathrm{~mA}$ for the first $30 \mathrm{~min}$. Participants were tested immediately before and after training and again $1 \mathrm{~h}$ later. Higher tDCS current was associated with increased performance for all test stimuli, but was greatest for repeated test stimuli with the presence of hidden-targets. This finding was replicated in a second set of subjects using a double-blind task design. Accuracy for target detection discrimination sensitivity ( $d^{\prime}$; $Z$ (hits) $-Z$ (false alarms)) was greater for $2.0 \mathrm{~mA}$ current (1.77) compared with $0.1 \mathrm{~mA}(0.95)$, with no differences in response bias $(\beta)$. Taken together, these findings indicate that the enhancement of performance with tDCS is sensitive to stimulus repetition and target presence, but not to changes in expectancy, mood, or type of blinded task design. The implications of these findings for understanding the cognitive mechanisms of tDCS are discussed.
\end{abstract}

Keywords: Learning, tDCS, Frontal Cortex, Object Recognition, Memory, Visual Search, Brain Stimulation

\section{Introduction}

TDCS involves the application of a weak DC electric current (usually 1-2 mA) to the scalp in order to modulate the activity of neurons in the brain (Pascual-Leone, Davey, Rothwell, Wassermann, \& Puri, 2002). In recent years, tDCS has garnered increasing interest for its application in the treatment of clinical disorders such as depression (Rigonatti et al., 2008), tinnitus (Fregni et al., 2006), and stroke (Webster, Celnik, \& Cohen, 2006). Other studies show that tDCS can produce cognitive enhancement in healthy subjects: TDCS has been shown to facilitate working memory (Fregni et al., 2006), motor learning (Antal et al., 2004; Galea \& Celnik, 2009; Reis et al., 2008, 2009), simple somatosensory and visual motion perception learning (Ragert, Vandermeeren, Camus, \& Cohen, 2008), and memory for word lists (Marshall, Molle, Hallschmid, \& Born, 2004).

We have previously reported that tDCS can increase learning in a complex visual search task involving the detection of target objects hidden in a virtual environment (Clark et al., 2012). In this study, participants were trained to detect target objects hidden in complex images of simulated environments and classify those images as target object present or target object absent. Participants received anodal tDCS during training, which was directed near the 10-10 EEG position F10 (over the right sphenoid bone lying above inferior frontal cortex). Large improvements in performance occurred in participants receiving tDCS using $11 \mathrm{~cm}^{2}$ electrodes during training. Similar to results presented by Iyer et al. (2005), these effects were dose-dependent, with performance improvement dependent upon current strength. Participants receiving 2.0 mAtDCS achieved accuracy scores significantly higher than those receiving 0.6 or 0.1 
mA, with a significant correlation found between changes in performance with learning and current strength.

Object detection is an important survival skill. Survival of humans and non-human animals as species has depended upon noticing camouflaged objects in the environment and reacting to

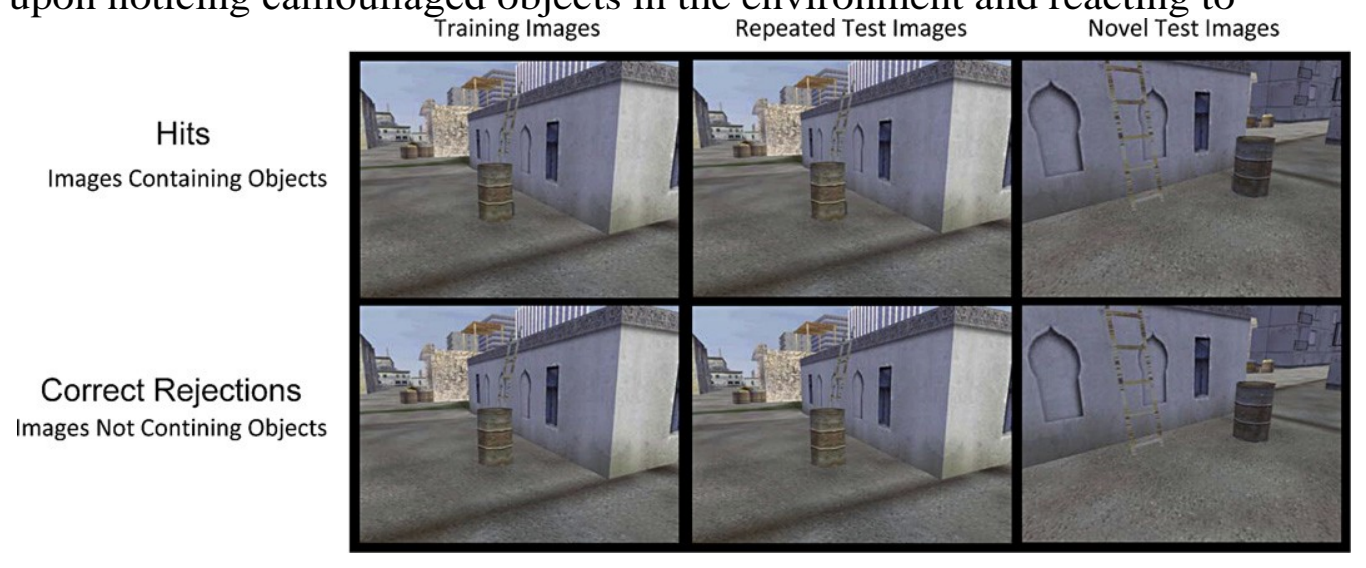

Fig. 1. Examples of training and testing stimuli. Test stimuli were either repeated from training (center panels) or novel, but similar to those viewed during training (right panels). Half of the images contained objects (upper panels), while the other half did not (lower panels). The target object is the rectangular gray object located behind the metal trash can, which indicates an explosive device.

hidden predators and food sources accordingly; therefore, hidden object detection has likely been an important factor in the evolution of human brain networks related to visual attention and perception (Clark et al., 2012). Furthermore, hidden threats to our safety are still present in the much more complex society we live in today, though they may be less prevalent than those we faced in our evolutionary past. One must avoid threats such as angry dogs, armed robbers, and moving vehicles, particularly in congested urban and suburban areas. Evidence of our evolutionary past in this context also can be found in modern video games and puzzles that exploit these innate survival mechanisms for enjoyment. Film makers also might add personal flare to their work by hiding objects or images (often referred to as "Easter eggs") that they hope viewers will dis- cover in a scene of their film. All of these examples underline the importance of brain networks that have evolved to aid in object detection in everyday life.

In the present investigation, we performed a re-analysis of data from Clark et al. (2012), examining the effects of stimulus novelty and target presence on test performance accuracy (referred to here as Experiment 1). In addition, we performed a replication experiment, using a new participant sample, with a double-blind, rather than single-blind protocol (Experiment 2) and optimized task design that balanced the numbers of novel and repeated test stimuli. We examined the main effects and interactions of tDCS current and image type on performance accuracy, target detection discrimination sensitivity ( $d^{\prime}$ ), and response bias $(\beta)$ during testing. We hypothesized that participants' responses to test images repeated between training and testing sessions would be more accurate than responses to test images not presented during training. We also investigated hypotheses related to the mechanisms of tDCS effects on learning and memory by examining the differential effects of tDCS on accuracy for repeated and novel images containing target objects (hits) as well as repeated and novel images not containing target objects (correct rejections).

\section{Materials and methods}

\subsection{Participants}

All participants met the following criteria: English as a first language, no history of head injuries or concussions resulting in loss of consciousness or hospitalization, right-handedness according to the Edinburgh Handedness Inventory (Oldfield, 1971), no history of psychiatric or neurological disorders, alcohol or drug abuse, or current medication affecting the CNS, and good or corrected vision and hearing. The study was approved by the Institutional Review Board of the University of New Mexico. The authors declare that they have no conflicts of interest in this research. 


\subsubsection{Experiment 1}

Thirty-nine healthy participants gave informed consent and participated in Experiment 1. Three participants with accuracy scores during the immediate test greater than two standard deviations below the mean ( 2 participants), or above the mean (1 participant) were excluded from analysis. Thus, the results from 36 participants (22 male, age $=24.0$ years, 4.9 years SD) were included in the analyses. Of these 36 participants, 13 received $2.0 \mathrm{~mA}$ tDCS, while the remaining 23 received $0.1 \mathrm{~mA}$.

\subsubsection{Experiment 2}

Twenty healthy participants gave informed consent and participated in Experiment 2. One subject with accuracy scores greater than two standard deviations below the mean was excluded from analysis. Thus, the results from 19 participants ( 11 male, age $=23.4$ years, 7.7 years SD) were included in analyses. Of these 19 participants, 9 received $2.0 \mathrm{~mA}$ tDCS, while the remaining 10 received $0.1 \mathrm{~mA}$.

\subsection{Target object detection task}

This experiment was designed in part to engage and maintain a typical participant's interest through its similarity to modern video games, which often include a wartime theme. Five-second video clips from training scenarios from the DARWARS virtual reality training environment were captured for use as feedback in the task (MacMillan et al., 2005). Six-hundred still images were extracted from these videos and edited to include or remove specific objects. Target objects that were hidden in these images included explosive devices concealed by or disguised as dead animals, roadside trash, fruit, flora, rocks, sand, or building structures, and enemies in the form of snipers, suicide bombers, tank drivers, or stone-throwers. For each of the images containing target objects, a corresponding image was created which did not contain a hidden target object. Thus, there were 1200 total images with $50 \%$ containing hidden target objects. Of these, 322 images, half containing concealed target objects, were selected for the learning task after review of the images by research associates unaware of the locations or defining features of specific target objects. The images were arranged in a random order and were not presented to participants in matched target object/no target object pairings. Examples of images presented during training and testing are presented in Fig. 1. Participants were allowed to stop if they found that the stimuli made them anxious or uncomfortable, although no one reported this or asked to end their participation in the study.

Participants were first tested for their baseline ability to detect target objects before training, after which participants were trained to detect target objects while receiving either $0.1 \mathrm{~mA}$ or $2.0 \mathrm{~mA}$ tDCS for $30 \mathrm{~min}$. Following training, participants were tested both immediately (immediate test) and 1 $\mathrm{h}$ after the end of the immediate test (1-h delay test, Fig. 2). Baseline, immediate, and 1-h delay tests consisted of 100 images presented with no feedback. Training sessions consisted of four 11-min blocks of 60 trials, each of which included an image and appropriate audiovisual feedback, with short rest periods between blocks. Each image was presented for $2 \mathrm{~s}$ with an inter-trial interval that varied across a range of values from 4 to $8 \mathrm{~s}$. Participants were instructed to scan the images for target objects with no prior information given about the nature of the target objects. Thus, the participant discovered the correct and incorrect responses to each image after examining the audiovisual feedback on each training trial. The feedback videos did not provide specific details of the shape or location of the target object, but enough information that the subject could infer the type and general position of the target object in the image and compare with their memory of the image. Four outcomes were possible: If a concealed target object was present in the image but was missed by the subject, the feedback movie showed the outcome, e.g., a sniper attack or bomb blast occurring, which the subject 
could use to infer the nature of the missed target object and then detect the same or similar target object on subsequent trials. At the same time,

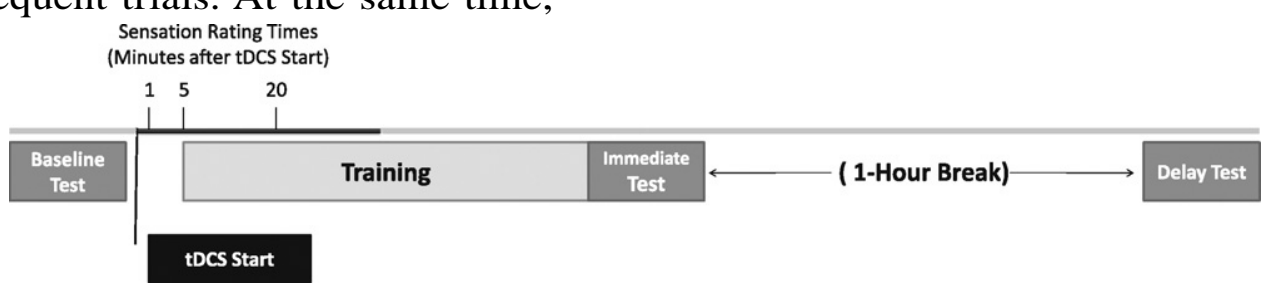

Fig. 2. Object detection training and testing paradigm. Training lasted approximately 1 h. Each test lasted about 15 min. TDCS was administered 5 min before training and lasted for a total of $30 \mathrm{~min}$. Participants were asked to indicate whether or not tDCS-induced sensation was present at three time points during administration. Participants performed baseline, immediate, and delay tests, each consisting of 100 still-image stimuli without feedback. Immediate and delay tests were separated by a 1-h break. Some images in the immediate test were repeated from training, while others were novel and had not been seen previously. Examples of stimuli can be found in Clark et al. (2012).

computer-generated voice-over indicated that the target object had been missed and there had been a casualty, but gave no specific information as to the identity of the target object. If a concealed target object was present and detected, the movie showed the scene progressing without harm and the voiceover complimented the subject for their performance. If a concealed target object was not present, and the subject incorrectly indicated that it was present, the voice-over chastised the subject for delaying the mission. Finally, when there was no concealed target object and the subject indicated this correctly, the voice-over praised the subject. Participants were instructed to respond as accurately as possible when making their responses to the stimuli. No instruction was given to indicate that response time would be a measure of performance, though subjects were asked to respond to each image within the 2-s display time. Importantly, a portion of the stimuli used in the immediate test had been presented during training, while the remaining stimuli were similar in content and had the same types of target objects, but had not been presented to the subject during training (Fig. 1). Thus, memory for trained images and the generalization of the training to novel images could be examined. The 1-h delay test was designed to examine retention of learned target object detection ability.

Eighteen percent of stimuli in the immediate test of Experiment 1 were repeated from training. This was incidental and not included as a part of the original study design. Following our analysis of the effect of test image type on performance accuracy, Experiment 2 was designed in which the immediate test stimuli contained 50\% repeated and 50\% novel stimuli, rather than $18 \%$ repeated and $82 \%$ novel in Experiment 1. In addition, a double-blind design was used for Experiment 2, rather than single-blind design as in Experiment 1. All other task-related procedures in Experiment 2 were identical to those in Experiment 1.

\subsection{Transcranial direct current stimulation}

Anodal tDCS was delivered for 30 min near 10-10 EEG location F10, over the right sphenoid bone. The location near F10 was suggested from functional magnetic resonance imaging (fMRI) and magnetoencephalography (MEG) studies of changes in brain networks associated with the acquisition of expertise in this task (Clark et al., 2012). TDCS was administered through $11 \mathrm{~cm}^{2}$ square salinesoaked sponge electrodes. The cathode was placed on the subject's left upper arm. Electrodes were secured to the scalp and upper arm using Coban self-adherent wrap. TDCS was initiated 5 min before training and continued throughout the first two of four training blocks (Fig. 2). We chose this timing because of the effects of tDCS demonstrated to occur after tDCS has ended in previous research (Antal et al., 2004). Current was set to either $0.1 \mathrm{~mA}$ or $2.0 \mathrm{~mA}$. A current strength of $0.1 \mathrm{~mA}$ (the lowest setting on our stimulation device) was selected as our control condition in order to induce physical sensation associated with tDCS (e.g. tingling and/or itching) without stimulating the brain areas beneath. Recent research has shown that traditional methods of "sham" tDCS stimulation involving ramping the current on and then off after a short (usually $30 \mathrm{~s}$ ) duration may not be as effective in blinding participants to stimulation condition as previously thought (Poreisz, Boros, Antal, \& Paulus, 2007). 
at the electrode size used in this study have no effect on brain activity in neural tissue $12 \mathrm{~mm}$ below the skin surface. In our control condition, participants received less than $20 \%$ of this current strength, so we are confident that this stimulation condition had little or no effect on the brain.

Participants, but not experimenters, were blind to tDCS current in Experiment 1 (single blind), while both participants and experimenters were blind to the current delivered in Experiment 2 (double blind). Experimenter blinding was accomplished using a coded switch box, with inputs for positive and negative leads from two current generators and outputs for only two electrodes, one anode and one cathode. One current generator was set to $0.1 \mathrm{~mA}$ and the other was set to $2.0 \mathrm{~mA}$. A six- way switch interrupted the circuit, with three settings supplying current to the output leads from one current generator, and the remaining three supplying the output from the other current generator. The inputs that were not actively supplying current to the output leads were routed through a simple circuit loop to maintain the activity of the inactive current generator. The six-way switch was coded by a third party to ensure experimenter blinding.

During tDCS, participants were asked to describe their physical sensations at 1, 5, and 20 min after the start of tDCS (Fig. 2). For Experiment 1, sensation data were recorded using a list of 10 descriptors including, in order of appearance: no sensation, cold sensation, some tingling, warm sensation, lots of tingling/some itching, very warm, lots of itching, burning (like a sunburn), burning (like scalding water), hurts a lot. TDCS was stopped if participants reported any of the last three descriptors, though no subjects in this study reached this criterion. In Experiment 2, subjects were asked to report sensation on three 10-point Likert scales for itching, tingling, and heat. TDCS was stopped if participants reported a seven or higher on any scale, though no one reported this level of sensation in this study.

\subsection{Data analysis}

We first compared participants' overall accuracy in detecting target objects in our initial study, as reported in Clark et al. (2012), to accuracy observed in the replication study (Experiment 2) using a $2 \times$ $2 \times 3$ repeated measures ANOVA, comparing experiment (Experiment 1 and Experiment 2), tDCS current level (0.1 mA and $2.0 \mathrm{~mA})$, and test time (baseline, immediate test, and delay test). The overall proportion of correct responses was used as a measure of accuracy for this analysis. Simple effects were corrected using Bonferroni adjustments of $\alpha=.025$ (.05/2) for simple effects of test and $\alpha=.017(.05 / 3)$ for simple effects of tDCS current. Individual contrasts between tests were Bonferroni-corrected at $\alpha=$ $.008(.05 / 6)$.

In our analysis of target detection discrimination sensitivity, we first compared signal detection (d') using a 3-way repeated measures ANOVA was used to compare experiment (Experiment 1 and Experiment 2), tDCS current level (0.1 mA and $2.0 \mathrm{~mA}$ ), and test time (baseline, immediate test, and delay test). We then compared participant's signal detection (d') scores for repeated and novel stimuli in the immediate test using a separate analysis. This $2 \times 2 \times 2$ repeated measures ANOVA compared d' between experiment (Experiment 1 and Experiment 2), tDCS current level (0.1 mA and $2.0 \mathrm{~mA}$ ), and image type (repeated and novel).

We also compared response bias $(\beta)$ between tDCS current levels using a $2 \times 3$ repeated measures ANOVA. This analysis compared test time (baseline, immediate test, and delay test) and tDCS current level (0.1 $\mathrm{mA}$ and $2.0 \mathrm{~mA})$ to determine the degree to which response bias changed throughout the task as a function of tDCS. Signal detection (d') scores as well as response bias $(\beta)$ scores reported in this analysis were calculated based on the hit (correct responses to images containing target objects) and false alarm (incorrect responses to images not containing target objects) rates according to calculations described by Stanislaw and Todorov (1999). Briefly, signal detection (d') is calculated by subtracting the $\mathrm{z}$-normalized false alarm rate from the z-normalized hit rate, while response bias $(\beta)$ is calculated by raising e to the power of $1 / 2$ the difference between the squared, $z$-normalized false alarm rate and the squared, z-normalized hit rate. Response bias $(\beta)$ scores were averaged across both experiments.

$$
d^{*}=Z \text { (Hits) }-Z \text { (false alarms) }
$$

$\beta=e \frac{(Z(\text { Hits }))^{2}-(Z(\text { false alarms }))^{2}}{2}$ 
In a more comprehensive analysis of our results from these two experiments, we divided our analysis of tDCS effects on learning into two separate analyses. The first of these analyses focuses on responses to images containing target objects, where accuracy is likely related to identifying the location of hidden target objects within the image (hits). The second analysis focuses on classification of images without target objects, where target object detection is not necessary and accuracy is based only on classification of the image (correct rejections). We examined the effects of tDCS on hits and correct rejections in images repeated from training and novel images in order to examine the effects of tDCS on object detection and image classification learning, respectively.

We compared the effects of tDCS on hits observed in Experiment 1 with those of Experiment 2 using a separate three-way ANOVA. This $2 \times 2 \times 2$ ANOVA compared accuracy for images containing hidden objects in the immediate test between experiment (Experiment 1 and Experiment 2), image type (repeated and novel), and tDCS current level (0.1 mA and $2.0 \mathrm{~mA}$ ). The proportion of correct responses to images in which an object was present was used as a measure of accuracy. All simple effects of image type and tDCS current were compared to Bonferroni corrected $\alpha$ of $0.025(.05 / 2)$.

Another $2 \times 2 \times 2$ ANOVA compared accuracy for images that did not contain a hidden target object (correct rejections) in the immediate test between experiment (Experiment 1 and Experiment 2), image type (repeated and novel), and tDCS current level (0.1 mA and $2.0 \mathrm{~mA})$. Correct rejections were used as a measure of accuracy in this analysis to examine the extent to which non-object-related image classification, rather than target object detection, was dependent on the within- and betweensubject variables.

The influence of sensation on accuracy in the immediate test was examined using two different methods, depending on the way the data was collected. Sensation data from Experiment 1 was treated as a binary variable (sensation present or sensation absent), given that the ratings were descriptors, not ordinal or interval level scales. Accuracy was contrasted between participants who did and did not report sensations using Student's $t$-test to determine the degree to which sensation influenced performance. Sensation data from Experiment 2 was analyzed using linear regression in order to determine the effect of tDCS-induced sensation on learning and performance in the task. Sensation ratings from all three measures (itching, heat, and tingling) were first entered into a stepwise multiple regression. When this model was determined non-significant, individual linear regression analyses were performed on each measure to determine the proportion of variance accounted for by each of the different measures of sensation.

We also compared mood data obtained before and after experimentation between tDCS groups using repeated measures ANOVAs to determine what, if any affect stimulation had upon the various self-reported measures of mood obtained in the study. Previous research by Barrett et al. (2004) has demonstrated that stimulation near the areas we targeted in this study can result in changes in mood and affect, although stimulation parameters in the study by Barrett and colleagues (i.e. cathode location, specific anode location, electrode size, and current strength) were somewhat different from those used in our research. Mood data was assessed by participants' self-report of their agreement with various statements, such as "I feel nervous or excited," on 6 point Likert scales, with 0 being "not at all” and 5 being "very much".

\section{Results}

\subsection{TDCS effects on learning and retention}

In this $2 \times 2 \times 3$ repeated measures ANOVA comparing overall accuracy between experiment (Experiment 1 and Experiment 2), tDCS current level (0.1 mA and $2.0 \mathrm{~mA}$ ), and test time (baseline, immediate test, and delay test), the three-way interaction between experiment, test, and current was not significant. No main effect or two way interaction involving experiment as an independent variable was identified in our analyses, indicating that the results of Experiment 2 were not significantly different from those of Experiment 1 (all $p$ s $>0.1$ ). Participants' performance increased significantly across the three test sessions in both experiments. Delay test scores were slightly, though significantly lower than immediate test scores, but participants performed significantly better than baseline in both of these tests (Fig. 3a). The degree to which performance increased with training, however, 
depended on tDCS current. Both $0.1 \mathrm{~mA}$ and $2.0 \mathrm{~mA}$ groups performed significantly better as training progressed, but participants receiving 2.0 mA tDCS performed significantly better than those receiving $0.1 \mathrm{~mA}$. Simple effects of current were significant for the immediate test and the delay test, but not for baseline performance (Fig. 3b), indicating that this effect was not due to baseline differences in performance. Descriptive and inferential statistics from this analysis can be found in Tables 1 and 2, respectively.

\section{Table 1}

Descriptive statistics (mean \pm standard error) for the effects of TDCS on learning and retention as measured by accuracy in the baseline, immediate, and delay tests, combined across Experiments 1 and 2.

\begin{tabular}{cccc} 
& Baseline & \multicolumn{2}{c}{ Immediate test } \\
$2.0 \mathrm{~mA}$ & $52.8 \pm 0.7 \%$ & $80.9 \pm 1.7 \%$ & $78.4 \pm 1.6 \%$ \\
$0.1 \mathrm{~mA}$ & $52.9 \pm 0.8 \%$ & $67.8 \pm 2.3 \%$ & $63.0 \pm 2.3 \%$ \\
Total & $52.9 \pm 0.5 \%$ & $73.1 \pm 1.7 \%$ & $68.3 \pm 1.8 \%$ \\
\hline
\end{tabular}

Table 2

Inferential statistics (degrees of freedom, $F$ statistics, and $p$ values) for the effects of TDCS and training on learning and retention, combined across Experiments 1 and 2.

\begin{tabular}{lccc}
\multicolumn{2}{c}{ Degrees of freedom } & F statistic & $p$ value \\
\hline Test (ME) & 2,100 & 125.377 & $7.73 \mathrm{e}^{-20}$ \\
Baseline vs. immediate (PC) & 1,51 & 177.170 & $3.21 \mathrm{e}^{-10}$
\end{tabular}

$\begin{array}{llll}\text { Baseline vs. delay (PC) } & 1,50 & 105.109 & 6.90 \mathrm{e}^{-14}\end{array}$

$\begin{array}{lccc}\text { Immediate vs. delay (PC) } & 1,50 & 27.914 & 2.77 \mathrm{e}^{-\mathrm{o}} \\ \begin{array}{lll}\text { tDCS current } \times \text { test (IE) } \\ \text { tDCS current (SE) }\end{array} & 2,100 & 15.075 & 4.82 \mathrm{e}^{- \text {工 }}\end{array}$

$\begin{array}{lccc}\text { Baseline } & 1,50 & 0.435 & 0.512 \\ \text { Immediate test } & 1,50 & 14.864 & 2.27 \mathrm{e}^{-4} \\ \text { Delay test } & 1,50 & 14.803 & 3.31 \mathrm{e}^{-4}\end{array}$


$\mathrm{ME}=$ main effect, $\mathrm{PC}=$ pair-wise contrast, $\mathrm{IE}=$ interaction effect, $\mathrm{SE}=$ simple effect. Simple effects were corrected using Bonferroni adjustments of $=.025(.05 / 2)$ for simple effects of test and $=.017(.05 / 3)$ for simple effects of tDCS current Individual contrasts between tests were Bonferroni-corrected at $\alpha=.008(.05 / 6)$.
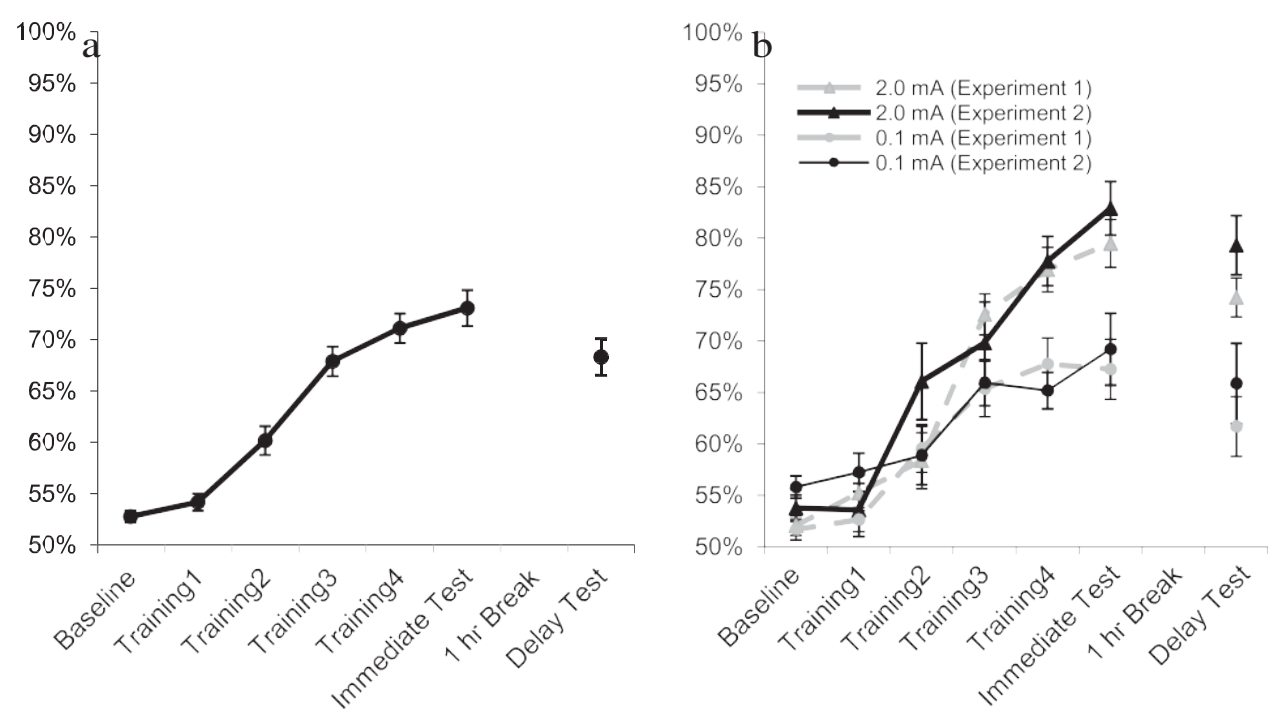

Fig. 3. Percentage of correct responses during different phases of training and testing. (a) Shows the effect of training. Participants' performance increased significantly with training across both experiments and remained significantly different from baseline following the 1-h break. (b) Shows the effect of current. Participants in Experiment 2 performed in a similar manner as those in Experiment 1 (broken lines). In both experiments, participants receiving $2.0 \mathrm{~mA}$ (triangles) significantly outperformed those receiving $0.1 \mathrm{~mA}$ (circles) in the immediate and delay tests. Rates of forgetting over the 1-h break were similar in both experiment.

\section{Table 3}

Signal detection (d') scores for the effects of image type and tDCS current on hidden object detection accuracy.

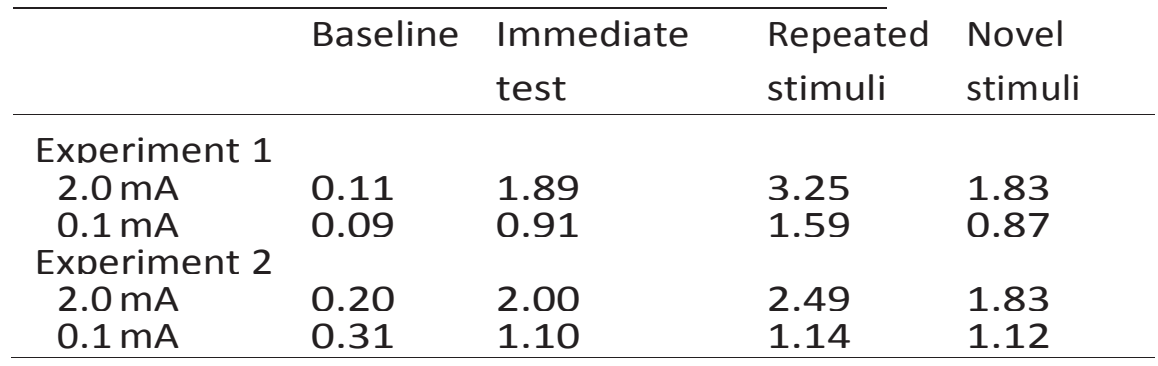




\subsection{TDCS effects on signal detection (d')}

In our analysis of signal detection, we first used a $2 \times 2 \times 3$ repeated measures ANOVA to compare $d$ ' between experiment (Experiment 1 and Experiment 2), tDCS current level (0.1 mA and $2.0 \mathrm{~mA}$ ), and test time (baseline, immediate test, and delay test). There were no significant differences in $d^{*}$ between experiments (all main and interaction effect $p$-values $>0.1$ ). Main effects were significant for tDCS current level $\left(F(1,49)=15.386, p=2.73 \mathrm{e}^{-4}\right)$ and test time $(F(2,98)=$ $\left.110.980, p=1.60 \mathrm{e}^{-18}\right)$, with greater $d$ ' scores for participants receiving $2.0 \mathrm{~mA}$ tDCS. There was a significant interaction between tDCS current level and test time $\left(F(2,98)=13.101, p=1.1 \mathrm{e}^{-4}\right)$, with significantly greater $d$ ' for participants in the $2.0 \mathrm{~mA}$ tDCS condition during the immediate test $\left(F(1,51)=15.567, p=2.44 \mathrm{e}^{-4}\right)$ and delay test $\left(F(1,49)=15.213, p=2.93 \mathrm{e}^{-4}\right)$, but not at baseline $(p>$ 0.1). Significant simple effects of test time were identified both for participants receiving $2.0 \mathrm{~mA}$ tDCS $\left(F(2,38)=135.690, p=1.34 \mathrm{e}^{-13}\right)$ and $0.1 \mathrm{~mA} \operatorname{tDCS}\left(F(2,60)=22.974, p=7.0 \mathrm{e}^{-6}\right)$, though signal detection scores increased after training and remained greater than 1 for the $2.0 \mathrm{~mA}$ groups, while $d$ ' scores remained below 1 for the duration of the experiment for those in the $0.1 \mathrm{~mA}$ condition. Signal detection $\left(d^{\prime}\right)$ scores for each condition can be found in Table 3.

In our second analysis of effects of tDCS on signal detection, we used a $2 \times 2 \times 2$ repeated measures ANOVA to compare $d$ ' between experiment (Experiment 1 and Experiment 2), tDCS current level $(0.1 \mathrm{~mA}$ and $2.0 \mathrm{~mA})$, and image type in the immediate test (repeated and novel). The main effect of experiment was nonsignificant in this analysis, as were the interaction of experiment and tDCS current level, and the three-way interaction (all $p$-values $>0.1$ ). The two-way interaction between experiment and image type was significant $(F(1,51)=4.771, p=0.034)$, although we believe that this is an artifact of the unequal proportions of repeated stimuli between the two experiments. Main effects were significant for tDCS current level $\left(F(1,51)=18.532, p=7.58 \mathrm{e}^{-5}\right)$ and image type $\left(F(1,51)=22.292, p=1.86 \mathrm{e}^{-5}\right)$, with greater $d^{\prime}$ scores for participants in the $2.0 \mathrm{mAtDCS}$ condition and greater $d$ ' scores for responses to repeated stimuli. The interaction between tDCS current level and image type was also significant $(F(1,51)=9.566, p=0.003)$, with significant simple effects of image type for participants receiving $2.0 \mathrm{~mA} \operatorname{tDCS}\left(F(1,20)=24.117, p=8.43 \mathrm{e}^{-5}\right)$, but not for participants receiving $0.1 \mathrm{~mA}(p>0.1)$. Simple effects of tDCS current level were present both for participants' responses to repeated $\left(F(1,51)=18.287, p=8.02 \mathrm{e}^{-5}\right)$ and novel stimuli $(F(1$, $51)=12.124, p=0.001)$. Signal detection ( $d$ ') scores for each condition can be found in Table 3. In our analysis of response bias, we compared 'between tDCS current level (0.1 mA and $2.0 \mathrm{~mA})$ and test time (baseline, immediate test, and delay test) using $2 \times 3$ repeated measures ANOVA. Data were combined across experiments for this analysis. All main and interaction effects of tDCS current were nonsignificant (all $p s>0.1$ ), though there was a main effect of test on response bias, with larger $\beta$ (greater tendency to respond "target absent") in the immediate test $(\beta=1.54)$ than at baseline $(\beta=1.08)$ or the delay test $(\beta=1.06)$.

\subsection{Effects of image type and tDCS current on classification of images containing objects (hits)}

In this analysis we performed a $2 \times 2 \times 2$ ANOVA comparing hits (accuracy for images containing hidden objects) in the immediate test between experiment (Experiment 1 and Experiment 2), tDCS current level (0.1 mA and 2.0 mA), and image type (repeated and novel). No main effect or three-way interaction involving experiment as an independent variable was identified in our analyses. Also, the two-way interaction between experiment and current was nonsignificant (all $p s>0.1$ ). There was a significant interaction between experiment and image type $(F(1,51)=4.095, p=0.048)$ in this analysis, with a slightly greater difference between repeated and novel stimuli in Experiment 1, though both simple effects of experiment were non-significant (all ps >0.1). Main effects of current (Fig. 4a) and image type (Fig. 4b) were statistically significant, indicating that participants were more accurate when receiving $2.0 \mathrm{~mA}$ tDCS, and when responding to repeated images. The two- way interaction between tDCS current level and image type was significant in this analysis as well. A simple effect of image type was present only in the $2.0 \mathrm{~mA}$ group, with responses to repeated images greater than those to 
novel. Simple effects of image type were not present in the $0.1 \mathrm{~mA}$ group. Simple effects of tDCS current were present for both repeated images and novel images which had not been seen previously (Fig. 4c). Descriptive and inferential statistics from this analysis can be found in Tables 4 and 5, respectively.

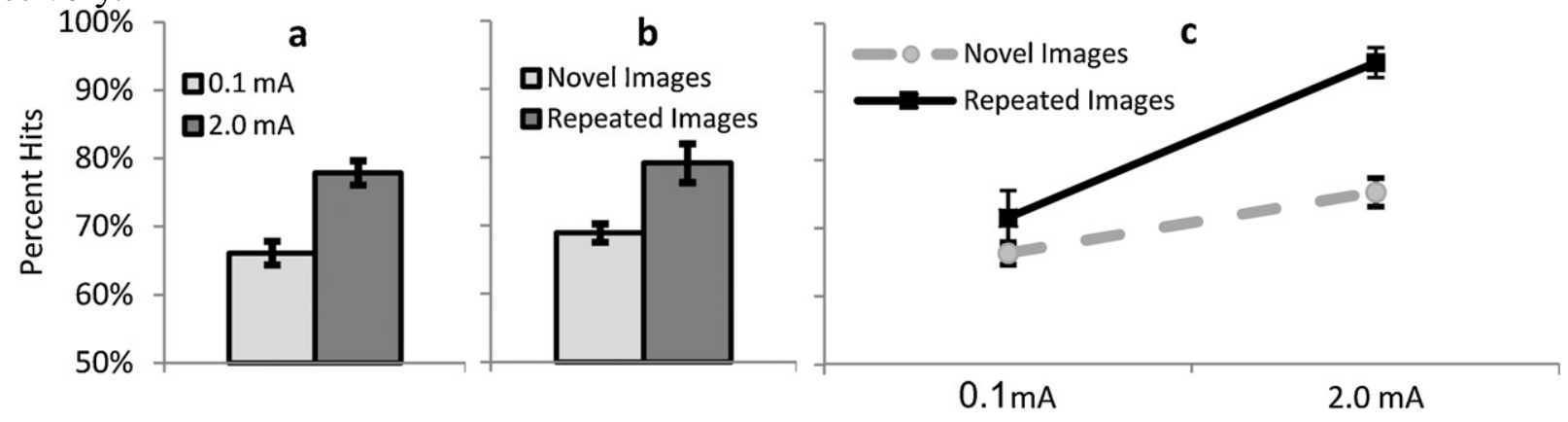

Fig. 4. Proportion of correct responses made for images containing objects the immediate test collapsed across both experiments. (a) Shows the effect of tDCS current. Participants accuracy increased significantly with tDCS current, with participants receiving the $2.0 \mathrm{~mA}$ (dark) outperforming those who received $0.1 \mathrm{~mA}$ (light). (b) Shows the effect of stimulus type for stimuli containing objects. Participants were significantly more accurate in detecting objects in stimuli repeated between training and test (dark) than in novel stimuli (light). (c) Shows the interaction of tDCS current and image type on accuracy for the immediate test. This interaction was significant, with greater tDCS effect on accuracy in detecting objects in repeated stimuli (solid line) than in novel stimuli (broken line).
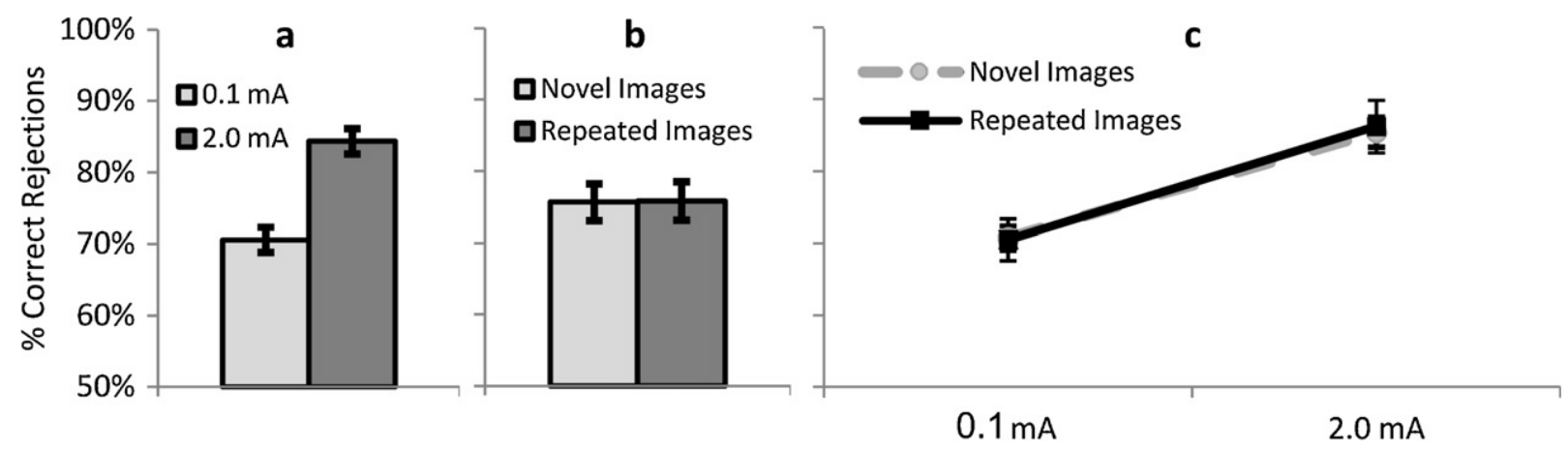

Fig. 5. Proportion of correct responses made for images that did not contain hidden objects during the immediate test collapsed across both experiments. (a) Shows the effect of tDCS current. Participants accuracy increased significantly with tDCS current, with participants receiving $2.0 \mathrm{~mA}$ (dark) outperforming those who received $0.1 \mathrm{~mA}$ (light). (b) Shows the effect of stimulus type. There was no difference in accuracy in detecting objects in repeated stimuli (dark) vs. novel stimuli (light). (c) Shows the interaction of tDCS current and image type on accuracy for the immediate test. This interaction was non-significant; with performance accuracy and tDCS effects being nearly identical for repeated stimuli (solid line) and novel stimuli (broken line).

\subsection{Effects of image type and tDCS current on classification of images without hidden objects (correct rejections)}

In this analysis we performed a $2 \times 2 \times 2$ ANOVAcomparing correct rejections (accuracy for images that did not contain a hidden object) in the immediate test between experiment (Experiment 1 and Experiment 2), tDCS current level $(0.1 \mathrm{~mA}$ and $2.0 \mathrm{~mA}$ ), and image type (repeated and novel). No main effect, two-way interaction, or three-way interaction involving experiment as an independent variable was identified in our analyses, indicating that training and tDCS effects in Experiment 2 are consistent with those of Experiment 1 for images without hidden objects (all $p s>0.1$ ). Main effects of current (Fig. 5a) were statistically significant in this analysis, as expected. Interestingly, no main effect (Fig. 5b), two-way interaction (Fig. 5c), or three-way interaction involving image type as an independent variable was identified in our analyses, indicating that image type did not affect image classification in images without hidden objects. Descriptive and inferential statistics from this analysis can be found in Tables 4 and 5, respectively.

\subsection{Effect of tDCS-induced sensation on performance}

All participants completed the training and testing phases of Experiment 1, however, sensation data were collected for only 18 of the 36 participants. For the 18 participants who completed the 
sensation questionnaire, there was no significant difference in accuracy (sensation present $65.0 \pm 4.7 \%$, sensation absent $71.3 \pm 3.7 \%, p>0.1$ ), indicating that sensation did not influence performance. Similarly, regression analyses indicated that there was no significant linear relationship present between sensation and accuracy in the immediate test in Experiment 2 for any of the three sensation measures collected, indicating that sensation did not significantly influence performance (all $p s>0.1$ ).

\section{Table 5}

Inferential statistics ( $F$ statistics and $p$ values) for the effects of image type and tDCS current on hidden object detection, combined across Experiments 1 and 2.

\section{Hitsb \\ tDCS current (ME) \\ Stimulus type (ME)}

Current $\times$ stimulus type (IE)

tDCS current (SE) Repeated stimuli

Novel stimuli

Stimulus type (SE)

$2.0 \mathrm{~mA}$

$0.1 \mathrm{~mA}$

Correct

tDCS Current

Stimulus type

(ME)

Current $x$

stimulus type (IE)

$$
F \text { statistic }{ }^{a}
$$

$1.17 \mathrm{e}^{-4}$

\section{E)}

7.219

17.820

10.762

\subsection{417}

48.008

0.934

7.334

0.034

0.009

0.854

$3.0 e^{-5}$

$9.71 \mathrm{e}^{-3}$

$1.0 e^{-4}$

$1.87 \mathrm{e}^{-3}$

. $p$ value

$9.96 \mathrm{e}^{-7}$

(18)

0.696

$\mathrm{ME}=$ main effect, $\mathrm{IE}=$ interaction effect, $\mathrm{SE}=$ simple effect. All simple effects were analyzed using Bonferroni adjustments of $\alpha=.025$ (.05/2).

a Degrees of freedom for all $F$ statistics were $(1,51)$.

b Accuracy for images containing objects, averaged across experiments.

c Accuracy for images not containing objects, averaged across experiments.

\subsection{Effect of tDCS on self-reported mood}

Mood data were collected for only 17 of the 36 participants in Experiment 1, but all 19 participants in Experiment 2. Results from the 36 participants for whom mood data was collected indicated that mood remained stable throughout the experiment, as signified by the nonsignificant interaction between time (before vs. after experimentation) and tDCS current in the majority of the measures collected (all ps >0.1). While an interaction was present between time and tDCS current for the measure nervousness/excitement $(F(1,35)=8.045 ; p=0.009)$ the change from before to after experimentation was similar between participants receiving $0.1 \mathrm{~mA}$ (mean change $=-1.7$ ) and $2.0 \mathrm{~mA}$ (mean change $=-0.8$ ). 


\section{Table 4}

Descriptive statistics (mean \pm standard error) for the effects of image type and tDCS current on hidden object detection accuracy.

\begin{tabular}{|c|c|c|c|c|c|c|}
\hline & \multicolumn{3}{|l|}{ Hitsa } & \multirow{2}{*}{$\begin{array}{l}\text { Correct } \\
\text { rejections } \\
\text { Repeated } \\
\text { stimuli }\end{array}$} & \multirow[b]{2}{*}{$\begin{array}{l}\text { Novel } \\
\text { stimuli }\end{array}$} & \multirow[b]{2}{*}{ Total } \\
\hline & $\begin{array}{l}\text { Repeated } \\
\text { stimuli }\end{array}$ & $\begin{array}{l}\text { Novel } \\
\text { stimuli }\end{array}$ & Total & & & \\
\hline $2.0 \mathrm{~mA}$ & $92.2 \pm 2.1 \%$ & $\begin{array}{l}74.0 \pm \\
2.0 \%\end{array}$ & $\begin{array}{l}77.9 \pm \\
1.8 \%\end{array}$ & $84.5 \pm 3.5 \%$ & $\begin{array}{l}84.1 \pm \\
3.5 \%\end{array}$ & $\begin{array}{l}84.4 \pm \\
2.6 \%\end{array}$ \\
\hline $\begin{array}{l}\text { u. I mA } \\
\text { Total }\end{array}$ & $\begin{array}{l}7 \cup .4 \pm 3 . y \% \\
79.1 \pm 2.8 \%\end{array}$ & $\begin{array}{l}\text { b5.ל } \pm \\
1.6 \% \pm \\
68.9 \pm \\
1.4 \%\end{array}$ & $1.7 \% \pm$ & $\begin{array}{c}69.8 \pm 2.5 \% \\
75.8 \pm 2.7 \%\end{array}$ & $\begin{array}{l}10 . \mathrm{U} \pm \\
2.5 \% \\
75.7 \pm \\
2.6 \%\end{array}$ & $3.5 \% \pm$ \\
\hline
\end{tabular}

a Accuracy for images containing objects, averaged across experiments.

b Accuracy for images not containing objects, averaged across experiments

\section{Discussion}

We have previously reported that anodal tDCS applied over right inferior frontal cortex facilitated learning to detect hidden objects in a dose-dependent manner (Clark et al., 2012). This main effect of tDCS on learning and performance was successfully replicated here in Experiment 2, using a doubleblind task design. We also attempt to answer a number of questions regarding what cognitive and perceptual changes might be induced by tDCS leading to increased performance in this task. In the analyses presented here, we examined the effects of both tDCS current and stimulus characteristics on accuracy in detecting objects and classifying images based on target object presence in order to further examine the mechanism of the effects of tDCS in these studies.

A number of hypotheses were examined regarding possible mechanisms of the effect of tDCS on learning and performance. First, accurate classification of images has been found to be related to similarity of previously learned images in previous studies (Medin \& Schaffer, 1978). Medin and Schafer's context theory suggested that this relationship is evidence of learning from exemplars in image classification, and that images that are similar to exemplars learned during training are more easily categorized. According to this hypothesis, participants should be more accurate in classifying images that are repeated from training, regardless of the presence of an object within the image. At the neural level, the learning of exemplars during training is dependent upon brain networks supporting the perception and encoding of stimuli (for a review see Paller \& Wagner, 2002). The physiological effects of anodal tDCS are thought to include increased excitability in the neocortex (Liebetanz, Nitsche, Tergau, \& Paulus, 2002), a theory that is supported by our recent findings of increased glutamatergic activity with anodal tDCS (Clark, Coffman, Trumbo, \& Gasparovic, 2011); therefore, it is possible that the anodal tDCS delivered in these experiments enhanced activity in specific brain regions, namely the right inferior frontal cortex, which may have facilitated the cognitive functions that support perception and encoding, leading to greater accuracy in classifying images later during testing.

Increased glutamatergic levels could have resulted in enhanced memory formation through a Hebbian mechanism in which cells become more readily active in a synchronous manner (Kelso, Ganong, \& Brown, 1986; Kirkwood \& Bear, 1994; Song, Miller, \& Abbott, 2000). A number of studies have shown that anodal tDCS is related to increased neural excitation through an effect on glutamatergic systems (Clark et al., 2011; Nitsche et al., 2003). Glutamatergic systems have been found to be important for learning (Bliss \& Collingridge, 1993). Therefore, tDCS may enhance learning through alteration of glutamatergic-associated neural plasticity, or some other affect on neurochemistry. Our analysis of object detection (hits) showed that participants were more accurate in detecting objects in images repeated between training and testing than in novel images; however, a significant interaction was found between tDCS current and stimulus repetition, indicating that the effects of tDCS on object detection performance (hits) were larger for repeated than novel test images. These results suggest that tDCS may facilitate plasticity in the brain networks responsible for encoding images, leading to a greater repertoire of exemplar images available to the participant during later testing. This hypothesis is contradicted, however, by data obtained for accuracy in classifying images that do not contain objects 
(correct rejections). TDCS increased participants' accuracy equally for repeated and novel test images that did not contain occluded target objects (correct rejections) indicating possible threats.

Another hypothesis is that tDCS over right inferior frontal cortex might specifically facilitate detection and learning of target objects indicating possible threats. While participants' accuracy was increased for images that were repeated from training, it is unlikely that this was due to explicit memory for stimulus type (e.g. object present or absent). Participants' accuracy for images not containing target objects (correct rejections) was not enhanced for images repeated from training. If participants remembered which specific images contained target objects and which did not, then one would expect this finding to generalize to all images, not simply those containing target objects. This hypothesis is supported by a number of prior studies, including our own (Clark et al., 2012) which found greater BOLD fMRI responses in right inferior frontal cortex evoked by occluded target objects indicating possible threats when contrasted with stimuli without target objects, suggesting that this brain region was a part of a network associated with the detection of threatening target objects. Additional studies have found that these regions are also involved in the perception of risk associated with decision making (Christopoulos, Tobler, Bossaerts, Dolan, \& Schultz, 2009).

One explanation for this pattern of results may be that greater tDCS enhancement of accuracy for repeated stimuli is due to scaling effects, in that tDCS may have less of an effect on performance for difficult (i.e. novel) stimuli than easy (i.e. repeated) stimuli. This would imply that tDCS would have a greater effect at an expert stage of performance when compared with a novice, because stimuli are more familiar at this stage. However, we have found that this tDCS protocol has a larger effect during the transition from novice to intermediate stages of learning when compared with subsequent intermediate to expert learning stages (Bullard et al., 2011). These findings would suggests that, rather than this tDCS protocol having a greater effects for the less difficult repeated stimuli compared with novel stimuli, the opposite was true, where tDCS showed a larger effect for stimuli at the novice stage when all stimuli are novel, compared with later stages of learning when stimuli have become more familiar.

Enhancing the excitability of the right frontal cortex may have also facilitated object detection performance due to enhanced perception or attention requisition during training, though these cognitive processes were not directly measured in these experiments. Posner and Petersen (1990) suggested that lateral frontal cortex is a key component of the fronto-parietal attention network, a brain network active in attention requisition during target detection. Greater attention requisition during visual search may lead to a greater probability of noticing target objects in the images, enhanced encoding of the location of the target object within the image and, therefore, greater accuracy. This hypothesis is therefore not exclusive of the hypothesis that tDCS enhanced memory for target object locations learned during training. Further studies are required to more precisely identify the effects of tDCS on attention and perception.

A final set of hypotheses regarding the possible effects of tDCS on cognition is related to experimenter bias based on stimulation condition (2.0 mA vs. $0.1 \mathrm{~mA})$. In this study, we examined bias by comparing single-blind with double-blind task designs. In the double-blind condition, neither the participant nor the experimenter were aware of the tDCS condition being used, and therefore could not have influenced the outcome. No significant differences were found between Experiment 1 using the single-blind design, and Experiment 2 using the double-blind design. Subjects in each of the two tDCS current groups $(0.1 \mathrm{~mA}$ and $2.0 \mathrm{~mA})$ performed similarly across the two experiments. These findings suggest that expectancies or changes in behavior of the experimenter did not significantly influence the outcome of the study.

Because tDCS causes sensation at the site of the electrodes (Furubayashi et al., 2008; Gandiga, Hummel, \& Cohen, 2006; Poreisz et al., 2007), we also examined the relationship between expectancy and behavioral effects through the examination of the relationship between skin sensation produced by tDCS and behavioral performance. This analysis tested the hypothesis that participants that experienced stronger somatic stimulation from tDCS would also expect to have larger behavioral effects, thus possibly influencing the test results. In Experiment 1, the performance of participants reporting sensation during tDCS was compared to that of participants who reported no sensation. As before (Clark et al., 2012), there was no significant difference in performance between groups. In Experiment 2, we used regression to look more quantitatively for effects of sensation. Again, we found that skin sensation was not the basis for increased learning and performance. These results suggest that the performance-facilitating effects of tDCS did not appear to be linked to skin sensation produced by the tDCS current in either experiment, nor to experimenter expectancy between single- and double-blind versions of the experiment.

One limitation of this study is the lack of precise control over the location of current flow through the brain. While we targeted the right frontal cortex, it is unlikely that tDCS resulted in focal 
stimulation of the brain, and may have included other brain regions as well. While there are no modeling studies that simulate the placement of the anode on the right frontal cortex with a cathode on the left upper arm, other studies indicate that even with two electrodes placed on the scalp the stimulation is diffuse and unpredictable (Datta et al., 2009; Miranda et al., 2009; Sadleir, Vannorsdall, Schretlen, \& Gordon, 2010; Wagner, Valero-Cabre, \& Pascual-Leone, 2007). Realistic, finite element head models suggest that a large fraction of the current passes into the brain via low resistance paths including the orbits and nose (Sadleir et al., 2010). Though there are no empirically based methods currently accepted to identify the precise path of tDCS current through the brain, magnetic resonance spectroscopy based methods to image tDCS induced changes in glutamatergic activity and other metabolites are currently being developed (Clark et al., 2011). This may help to better understand the brain networks and cognitive functions most affected by tDCS.

\section{Conclusion}

In summary, tDCS produced a significant increase in the accuracy of image classification and hidden target object detection after training, which was not related to the amount of skin sensation and was not different between single- and double-blind experimental designs. The effect of tDCS was greatest for test images containing target objects (hits) that were presented during training and again during testing, although tDCS also facilitated detection of target objects hidden in novel test images and classification of images without hidden target objects (correct rejections). The replication of these effects across experiments further supports the validity of these effects. These results indicate that the cognitive effects of tDCS interact with conditions of stimulus repetition and target object presence. These effects may be related in turn to the alteration of some combination of attentional, perceptual, visual search, memory encoding, or memory retrieval processes by tDCS. Additional studies are needed in order to more precisely under- stand the specific neural and cognitive mechanisms by which tDCS influences learning and performance.

\section{Acknowledgements}

This work was supported by The Defense Advanced Research Projects Agency (Government contract NBCHC070103) and the Department of Energy (Government contract DEFG02-99ER62764). Special thanks to Elaine Raybourn, Kyle Kenny, Neal Miller, Ron Denny and Alan Rolli, Jeremy Bockholt, Elizabeth Browning, Michael Doty, Megan Schendel, Dae Il Kim, Josef Ling, Jing Xu, Mark Skully, Jill Fries, Arvind Caprihan, Claudia Tesche, Sergey Plis, Diane Oyen, Blake Anderson and Francesca McIntire for help in experiment development, data collection and data analysis. The views, opinions, and/or findings contained in this article are those of the authors and should not be interpreted as representing the official views or policies, either expressed or implied, of the Defense Advanced Research Projects Agency, the Department of Defense, or the Department of Energy.

\section{References}

Barrett, J., Della-Maggiore, V., Chouinard, P. A., \& Paus, T. (2004). Mechanisms of action underlying the effect of repetitive transcranial magnetic stimulation on mood: behavioral and brain imaging studies. Neuropsychopharmacology, 29, 1172-1189.

3M Coban self-adherent wrap. (2012). [Apparatus]. http://www.3m.com.

Antal, A., Nitsche, M. A., Kincses, T. Z., Kruse, W., Hoffmann, K. P., \& Paulus, W. (2004). Facilitation of visuo-motor learning by transcranial direct current stimulation of the motor and extrastriate visual areas in humans. European Journal of Neuroscience, 19, 2888-2892.

Bliss, T. V. P., \& Collingridge, G. L. (1993). A synaptic model of memory: Long-term potentiation in the hippocampus. Nature, 361, 31-39.

Bullard, L., Browning, E., Clark, V., Coffman, B. A., Garcia, C., Jung, R., et al. (2011). Transcranial direct current stimulation's effect on novice versus experienced learning. Experimental Brain Research, 213, 9-13.

Christopoulos, G. I., Tobler, P. N., Bossaerts, P., Dolan, R. J., \& Schultz, W. (2009). Neural correlates of value, risk, and risk aversion contributing to decision making under risk. Journal of Neuroscience, 26, 6469-6472.

Clark, V. P., Coffman, B. A., Mayer, A. R., Weisend, M. P., Lane, T. D. R., Calhoun, V. D., et al. 
(2012). TDCS guided using fMRI significantly accelerates learning to identify concealed objects. NeuroImage, 59, 117-128.

Clark, V. P., Coffman, B. A., Trumbo, M. C., \& Gasparovic, C. (2011). Transcranial direct current stimulation (tDCS) produces localized and specific alterations in neurochemistry: A $1 \mathrm{H}$ magnetic resonance spectroscopy study. Neuroscience Letters, 500, 67-71.

Datta, A., Bansal, V., Diaz, J., Patel, J., Reato, D., \& Bikson, M. (2009). Gyri-precise head model of transcranial DC stimulation: Improved spatial focality using a ring electrode versus conventional rectangular pad. Brain Stimulation, 2, 201-207.

Fregni, F., Boggio, P. S., Lima, M. C., Ferreira, M. J., Wagner, T., \& Rigonatti, S. P. (2006). A shamcontrolled, phase II trial of transcranial direct current stimulation for the treatment of central pain in traumatic spinal cord injury. Pain, 122, 197-209.

Furubayashi, T., Terao, Y., Arai, N., Okabe, S., Mochizuki, H., \& Hanajima, R. (2008). Short and long duration transcranial direct current stimulation (tDCS) over the human hand motor area. Experimental Brain Research, 185, 279-286.

Galea, J. M., \& Celnik, P. (2009). Brain polarization enhances the formation and retention of motor memories. Journal of Neurophysiology, 102, 294-301.

Gandiga, P. C., Hummel, F. C., \& Cohen, L. G. (2006). Transcranial DC stimulation (tDCS): A tool for double-blind sham-controlled clinical studies in brain stimulation. Clinical Neurophysiology, 117, 845-850.

Iyer, M. B., Mattu, U., Grafman, J., Lomarev, M., Sato, S., \& Wassermann, E. M. (2005). Safety and cognitive effect of frontal DC brain polarization in healthy individuals. Neurology, 64, 872-875.

Kelso, S. R., Ganong, A. H., \& Brown, T. H. (1986). Hebbian synapses in hippocampus. PNAS, 83, 5326-5330.

Kirkwood, A., \& Bear, M. F. (1994). Hebbian synapses in visual cortex. Journal of Neuroscience, 14, 1634-1645.

Liebetanz, D., Nitsche, M. A., Tergau, F., \& Paulus, W. (2002). Pharmacological approach to the mechanisms of transcranial DC-stimulation-induced after- effects of human motor cortex excitability. Brain, 125, 2238-2247.

MacMillan, J., Tomlinson, R., Alexander, A. L., Weil, S. A., Littleton, B., \& Aptima, I. (2005). DARWARS: An architecture that supports effective experiential training. DARWARS Research Papers, http://www.darwars.com/downloads/2005\%20IITSEC\%20White\%20Paper\%20v2.pdf

Marshall, L., Molle, M., Hallschmid, M., \& Born, J. (2004). Transcranial direct current stimulation during sleep improves declarative memory. Journal of Neuroscience, 24, 9985-9992.

Medin, D. L., \& Schaffer, M. M. (1978). Context theory of classification learning. Psychological Review, 85, 207-238.

Miranda, P. C., Faria, P., \& Hallett, M. (2009). What does the ratio of injected current to electrode area tell us about current density in the brain during tDCS? Clinical Neurophysiology, 120, 1183-1187.

Nitsche, M., Fricke, K., Henschke, U., Schlitterlau, A., Liebetanz, D., Lang, N., et al. (2003). Pharmacological modulation of cortical excitability shifts induced by transcranial direct current stimulation in humans. The Journal of Physiology, 553, 293-301.

Oldfield, R. C. (1971). The assessment and analysis of handedness: The Edinburgh inventory. Neuropsychologia, 9, 97-113.

Paller, K. A., \& Wagner, A. D. (2002). Observing the transformation of experience into memory. Trends in Cognitive Sciences, 6(2), 93-102.

Pascual-Leone, A., Davey, N., Rothwell, J., Wassermann, E. M., \& Puri, B. K. (2002). Handbook of transcranial magnetic stimulation. New York: Oxford.

Poreisz, C., Boros, K., Antal, A., \& Paulus, W. (2007). Safety aspects of transcranial direct current stimulation concerning healthy participants and patients. Brain Research Bulletin, 72, 208-214.

Posner, M. I., \& Petersen, S. E. (1990). The attention system of the human brain. Annual Review of Neuroscience, 13, 25-42.

Ragert, P., Vandermeeren, Y., Camus, M., \& Cohen, L. G. (2008). Improvement of spatial tactile acuity by transcranial direct current stimulation. Clinical Neurophysiology, 119, 805-811.

Reis, J., Robertson, E., Krakauer, J. W., Rothwell, J., Marshall, L., \& Gerloff, C. (2008). Consensus: Can transcranial direct current stimulation and transcranial magnetic stimulation enhance motor 
learning and memory formation? Brain Stimulation, 1, 363-369.

Reis, J., Schambra, H. M., Cohen, L. G., Buch, E. R., Fritsch, B., \& Zarahn, E. (2009). Noninvasive cortical stimulation enhances motor skill acquisition over multiple days through an effect on consolidation. PNAS, 106, 1590-1595.

Rigonatti, S. P., Boggio, P. S., Myczkowski, M. L., Otta, E., Fiquer, J. T., \& Ribeiro, R. B. (2008). Transcranial direct stimulation and fluoxetine for the treatment of depression. European Psychiatry: Journal of the Association of European Psychiatrists, 23, 74-76.

Sadleir, R. J., Vannorsdall, T. D., Schretlen, D. J., \& Gordon, B. (2010). Transcranial direct current stimulation (tDCS) in a realistic head model. NeuroImage, 51, 1310-1318.

Song, S., Miller, K. D., \& Abbott, L. F. (2000). Competitive Hebbian learning through spike-timing dependent synaptic plasticity. Nature Neuroscience, 3, 919-926.

Stanislaw, H., \& Todorov, N. (1999). Calculation of signal detection theory measures. Behavior Research Methods, Instruments, \& Computers, 31(1), 137-149.

Wagner, T., Valero-Cabre, A., \& Pascual-Leone, A. (2007). Noninvasive human brain stimulation. Annual Review of Biomedical Engineering, 9, 527-565.

Webster, B. R., Celnik, P. A., \& Cohen, L. G. (2006). Noninvasive brain stimulation in stroke rehabilitation. NeuroRx, 3, 474-481. 